\title{
Introduction to Optical Fiber Communication in Lightwave Systems
}

\author{
Harminder Singh \\ Research Scholar, \\ E.C.E. Dept., \\ A.C.E.T., Amritsar
}

\author{
Atul Mahajan \\ H.O.D., E.C.E. Dept., \\ A.C.E.T., Amritsar
}

\begin{abstract}
From the last 10-15 years Optical Fibers are replacing the conventional ways of data transmission i.e. wires, where an optical fiber is a transparent, hair like cylindrical structure which carries data in form of light signals. As the number of users is growing day by day so as the transmission data, in order to accumulate this high amount of data the traditional wires was not an good option because with the enlargement of users, their needs also expand. One of the need of users is speed, although the wires were transmitting data but the speed was low with other losses in the transmission. Optical fiber transmits data in the form of light, so naturally the speed of data is $3^{*} 10^{8} \mathrm{~m} / \mathrm{s}$ (speed of light). In modern world almost everything will rely on optical cabels like that of telephone, television, internet lines etc. This paper focuses on the implementation of the optical network in metro span area that with what parameters, components, losses and efficiency an network working on light signal will handle the large amount of an metropolitan area.
\end{abstract}

\section{Keywords}

Network Evolution, Services, WDM, Impairments.

\section{INTRODUCTION}

With the use of optical fibers in commercial as well as in domestic sectors the load on the effective development of overall transmission increases. Transmission system capability has grown more faster as compared to the switching techniques. In metro connectivity to handle the new upcoming services advanced multiplexing techniques are needed, although WDM (Wavelength Division Multiplexing) exists but it will perform efficiently till the number of users are in its limit. Also with the development of SONET/SDH (Synchronous Optical Networking/Synchronous Digital Hierarchy) the architecture the metropolitan area network matures. The factors which degrades the performance of metro networks are optical signal-to-noise ratio (SNR), chromatic dispersion, distortions due to multiplexer/demultiplexer (MUX/DMUX), polarization mode dispersion (PMD), polarization dependent loss (PDL), transport layer impairments like amplifier noise, optical crosstalk, chirping etc . A number of commercial software packages have been originated to handle and design the fundamental base of MAN, they also helps in the analysis of transmission impairments and increases the transparency in the network, after the study of impairments a suitable network can be laid down which can provide sufficient efficiency for the metro networks.

\section{NETWORK EVOLUTION}

From the past 2 decades the load and architecture of networks has changed drastically. In present scenario besides of data transmission there is existence of images, movies and audiovideo calls. In early stages data was transmitted on time sharing basis but now the time has changed, now the data is to be transferred at any time at any place of the globe.

Accumulation of the high data is possible with the introduction of IP over network and IP over mobile. In the past there was IP over network but with the increase in needs and services IP over mobile originates which simply is the communication of media via mobile networking. Traffic is growing on the transmission media at a very high rate and in order to maintain the balance between data and users a large amount of bandwidth is needed.

Bandwidth is the amount of data that a transmission media can handle. With the increase in users bandwidth requirement is also growing at the same rate. The drawback in digital transmission is the availability of limited bandwidth as a small diameter wire can handle a limited amount of data at a time.

This drawback is resolved by introducing multiplexing techniques in fibers [1] [2], as data from different PONs (Passive Optical Networks) is multiplexed/joined together and is allowed to move along the same path thus saving the bandwidth and producing more space for newer data. Where PONs are the smaller optical networks which upon joining gave rise to a metro network

\section{SERVICES AND ARCHITECTURE}

Metro networks provide following services :-

\subsection{High Speed Internet Services (HSIS)}

As in the modern world entertainment goes on side by side with the work. Video calling, sending messages over different applications, accessing various sites, downloading movie/video clips, sending e-mails etc requires an fast internet connection. Then an another feature called as video-ondemand is there which enables an user to save an video and browse to it later.

\subsubsection{Storage Capacity}

IT Sector and governments require a large amount of storage capacity to make all the stuff at a single place. These sectors will focus on creating their own private networks for the guaranteed efficiency as their need of safer data is much more important than that of the residential needs. Remote database access and Intranet applications will be the primary area of focus for these organizations.

\subsection{Other services related with IP}

In order to cope up with the growing needs of users an metro network must be fulfilling the requirement of IP network services also. There are three types of IP services :- [3]

\subsubsection{Layer -2 Packet Services}

It is basically a choice which supports heavy amount of data and applications. It is a connection of virtual-wires interconnecting in an network. Switch interconnections can 
also be made through this service, that can be useful in the functioning of the big business links and government networks. Some examples of this service are ATM (Asynchronous Transfer Mode) machines, Frame Relay Networks, Ethernet etc.

\subsubsection{TDM Services}

This service provides security as well as efficiency to the metro network. It lowers the jitter possibility, where jitter is the worst case in the transmission of data, it is the highest level of ISI (Inter Symbol Interference). This service will add guarantee reception of information as there will be time slots for each data packet and wait mechanism for the adequate flow.

\subsubsection{Wave Services}

It is linked with DWDM (Dense Wavelength Division Multiplexing). This service meets with the very high bandwidth requirement and also adds bit-rate transparency to the system. Probability of BER (Bit Error Rate) and ISI is little more in this service as the message is compressed more than WDM.

\subsection{Architecture}

The architecture of an metro network must provide an assurance to the information from sender to destination side. For this the architecture includes :-

\subsubsection{Double Optical Connections}

Providing double optical connections between sender and receiver is termed as $1+1$ protection terminology. In this if the primary connection fails due to overload or may be because of internal/external parameters then a secondary connection should be there to continue the communication [4].

Optical reconfiguration is not required for the secondary connection between the nodes which is one of the major advantages of using two fibers as this will save the time of configuration already done on the primary fiber. The primary configurations are transferred to the secondary for the fast and reliable communication [5], this terminology also provides rapid recovery as data is not lost in case of failure of primary optical fiber.

\section{WDM}

WDM is a phenomenon of combining a number of wavelengths onto a single optical fiber. Problem of interchannel interference is removed thats why communication industry is going on large-scale transformation to bring T.V., Internet and Telecommunication on optical transmission in order to benefit from high speed and most importantly from large data rate to be provided by WDM.

WDM demolishes the huge opto-electronic bandwidth mismatch [6] by multiplexing different WDM channels from various users onto a single optical fiber but the condition is that they all should operate at a common electronic rate to provide synchronization to the incoming signal. In the transmission the signal comprises of a multiple nonoverlapping WDM ongoing signals.

There is an advance version of WDM that is DWDM, in that the channel spacing is reduced to an extent that they do not overlap i.e. channels are placed more closer is DWDM. A mux is used to collect and combine all the received channels from different PONs into a single wavelength and dmux performs the function of extracting all the available and received channels.

\section{IMPAIRMENTS}

Impairments or the losses in the optical fiber communication are basically of 2 types linear and non-linear impairments. Where linear impairments are associated with the low power of cw (continuous wave) laser and with higher power the non-linear losses are introduced, a threshold value is determined prior the signal transmission and if the power goes beyond that value then the non-linear impairment is observed.

Crosstalk, signal amplification, amplifier noise, filter concatenation etc are some of the examples of linear losses whereas the non-linear includes SPM (Self Phase Modulation), FWM (Four Wave Mixing), XPM (Cross Phase Modulation), Stimulated Brillouin Scattering, Stimulated Raman Scattering etc.

When user A wants to connect to user B but instead of user B, user $\mathrm{C}$ is connected then this type of phenomenon is referred to as crosstalk. It is also not advisable to amplify the light signal at each and every intermediate node as it adds losses to the system.

When light signal travels from different refractive indexes of the optical fiber then SPM is seen whereas FWM occurs when two or more light signals produce two new signals at the receiver side due to similar characteristics. Stimulated Brillouin Scattering and Stimulated Raman Scattering are associated with the photons and similar energy levels of the optical fiber surface respectively.

\subsection{Amplifier Effects}

EDFA (Erbium Doped Fiber Amplifier) [7] is a amplifier used in the amplification of the optical signal. It simply raises the power level of the optical signal irrespective of the amplification induced noise i.e. it does not removes noise but amplifies noise also with the light signal, which is not required. It amplifies only when the channel bandwidth is less than the EDFAs bandwidth.

\subsection{Chirping Effect}

In order to transmit the signal over the globe it has to travel 100 of $\mathrm{km}$, for this power of the light signal must be so adequate that it should not diminish at any point of the transmission media. But the power must not be increased beyond the pre-defined threshold value, if power gains an magnitude higher than the threshold value the this type of losses can originate. Under this frequency of signal varies.

\subsection{Crosstalk}

In an multimode fiber there are hundreds of light signals and there is an possibility that many of them can have same frequency levels. Signals with smaller difference in the frequencies can overlap with each other and the information may be distorted. In traditional coaxial cables this effect is common as in telephone lines one user can hear other user's voice. This can cause severe performance degradation [8].

\subsection{Other Effects}

Linear and non-linear effects are in majority at optical levels. But maintaining proper light levels can reduce their effects [9]. An analytical model can be used to calculate the penalty levels [10]. 


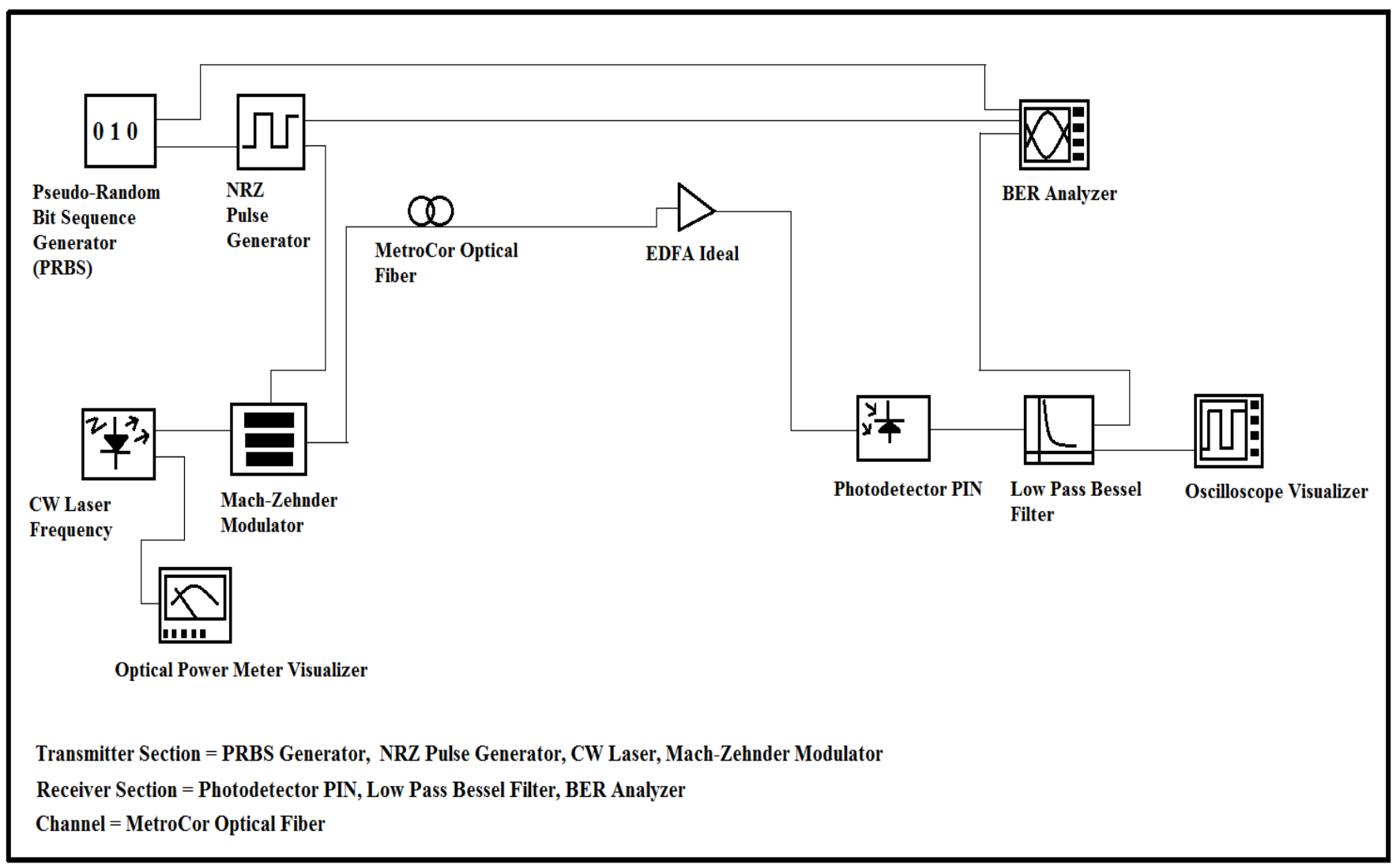

Fig. 1: Architectural Representation of Lightwave System.

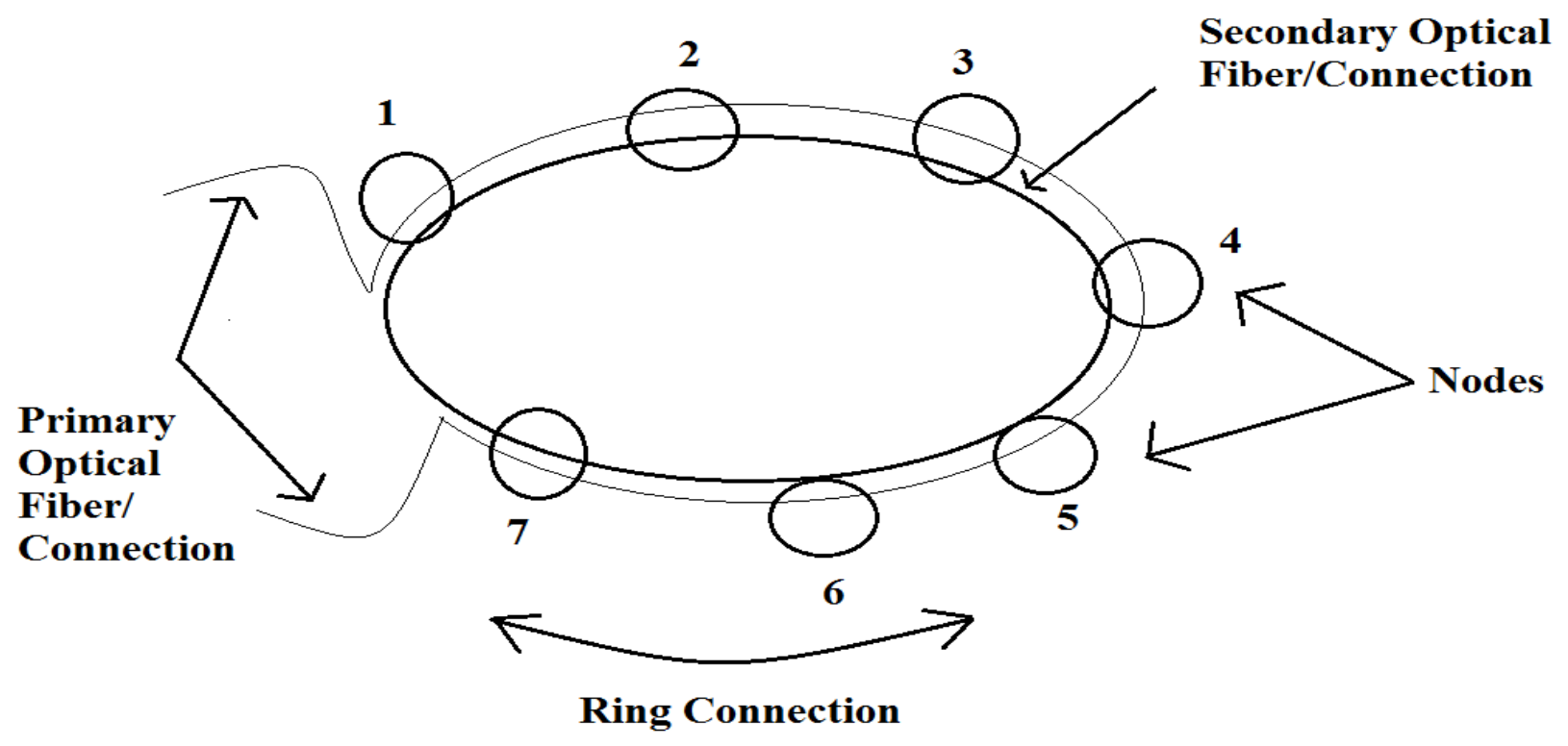

Fig. 2: Ring Connection of 2 Optical Fibers/1+1 Connection. 


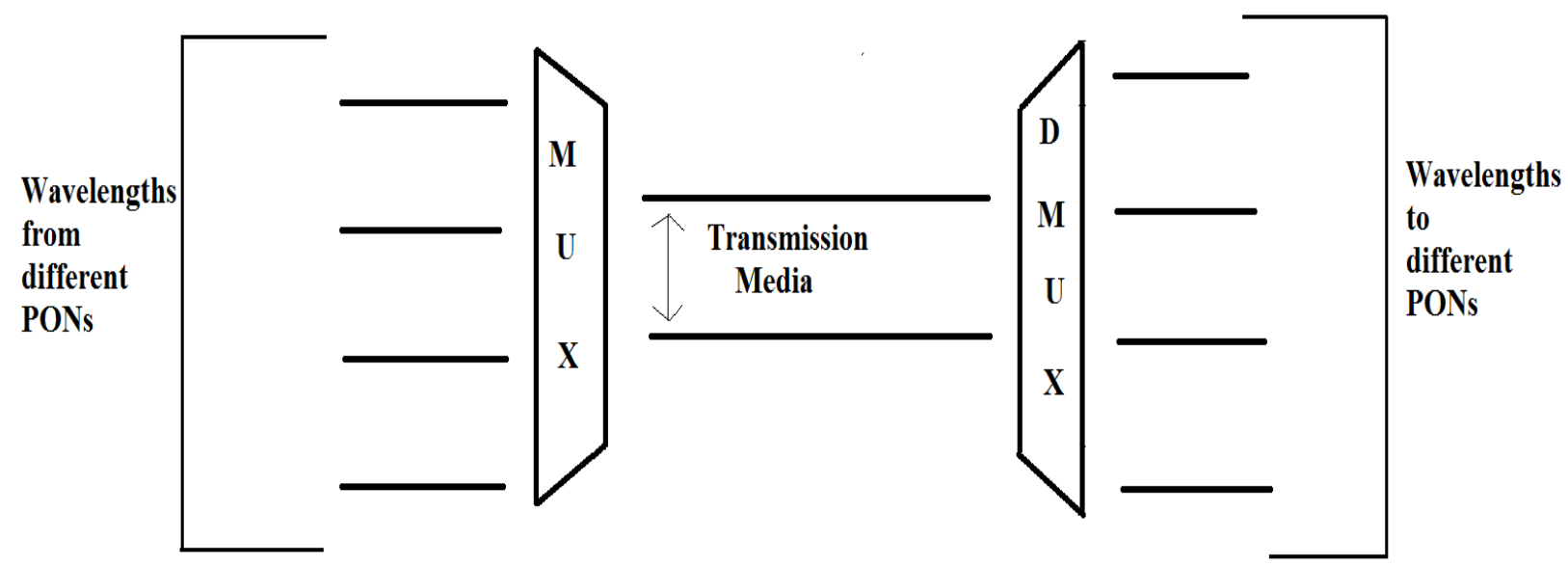

Fig. 3: WDM Architecture

\section{CONCLUSION}

Realization of a metro network needs high bandwidth, higher internet speed and a big data storage capacity. The surge in data messages and falling more in voice and video messages has created the need of the MAN. Configurable network can be implemented to control the panel of connections to handle the advanced dynamic applications. Computer simulations will be helpful in implementing the metro network and its topologies. Whatever direction the network follows the need is to follow that route and establish a trade off between users and the data. To meet this growing demand more advancements is needed in the existing network configurations. WDM and TDM can be bridged for this task. Designing such networks along with the high efficiency, reliability and faithful operation is an critical task as the WDM is a already established configuration for a limited number of users with packed bandwidth.

\section{FUTURE SCOPE}

Data rate can be increased with the help of symmetric compensation techniques, power can be saved and distance of light transmission can be increased. Quality factor and bit error rate are inversely proportional to each other, so the quality factor or the efficiency of the overall lightwave system can be raised by altering the various parameters like that of the power and frequency of continuous wave laser, negative dispersion of metrocor optical fiber, cutoff frequency of low pass bessel filter etc. All the parameters are interlinked to each other so a optimized variation can be performed to enhance the quality factor but to a adequate value.

\section{REFERENCES}

[1] I. Djordjevic, B. Vasic, M. Ivkovic, and I. Gabitov, "Achievable information rates for high-speed long-haul optical transmission," J. Lightw. Technol., vol. 23, no. 11, pp. 3755-3763, Nov. 2005.

[2] R.-J. Essiambre, G. J. Foschini, G. Kramer, and P. J. Winzer, "Capacity limits of information transport in fiber-optic networks," Phys. Rev. Lett., vol. 101, p. 163901, 2008.

[3] Joseph Berthold, Senior Member, IEEE, Adel A. M. Saleh, Fellow, IEEE, Fellow, OSA, Loudon Blair, Member, IEEE, and Jane M. Simmons, Senior Member, IEEE Optical Networking: Past, Present, and Future Journal Of Lightwave Technology, vol. 26, NO. 9, MAY 1,2008

[4] J. M. Simmons, Optical Network Design and Planning. Norwell, MA: Springer, 2008

[5] Kazuro Kikuchi, "Fundamentals of Coherent Optical Fiber Communications" Journal of Lightwave Technology, vol. 34, no. 1, January/February 2016.

[6] S. J. B. Yoo, Senior Member, IEEE, "Wavelength Conversion Technologies for WDM Network Applications", Journal Of Lightwave technology, vol. 14, no. 6 , june 1996 .

[7] P. C. Becker, N. A. Olsson, and J. R. Simpson, ErbiumDoped Fiber Amplifiers, Academic Press, San Diego, 1998.

[8] K. P. Ho, "Analysis of homodyne crosstalk in optical networks using Gram-Charlier series," J. Lightwave Technol., vol. 17, pp. 149-153, 1999.

[9] N. Antoniades, M. Yadlowsky, and V. L. daSilva, "Computer simulation of a metro WDM ring network," IEEE Photon. Technol. Lett., vol. 12, no. 11, pp. 15761578, Nov. 2000.

[10] N. Antoniades, A. Boskovic, I. Tomkos, N. Madamopoulos, M. Lee, I. Roudas, D. Pastel, M. Sharma, and M. Yadlowsky, "Performance engineering and topological design of metro WDM optical networks," IEEE J. Select. Areas Commun. (Special Issue on WDM Based Optical Networks), vol. 20, pp. 149-165, Jan. 2002. 